\title{
Statistical correlations in an ideal gas of particles obeying fractional exclusion statistics
}

\author{
F. M. D. Pellegrino, ${ }^{1}$ G. G. N. Angilella, ${ }^{2, *}$ N. H. March, ${ }^{3,4}$ and R. Pucci ${ }^{2}$ \\ ${ }^{1}$ Scuola Superiore di Catania, via S. Nullo, 5/i, I-95123 Catania, Italy \\ ${ }^{2}$ Dipartimento di Fisica e Astronomia, Università di Catania, I-95123 Catania, Italy, CNISM, Università di Catania, I-95123 Catania, \\ Italy, and INFN, Sezione Catania, via S. Sofia, 64, I-95123 Catania, Italy \\ ${ }^{3}$ Department of Physics, University of Antwerp, Groenenborgerlaan 171, B-2020 Antwerp, Belgium \\ ${ }^{4}$ Oxford University, Oxford, England
}

(Received 10 August 2007; published 20 December 2007)

\begin{abstract}
After a brief discussion of the concepts of fractional exchange and fractional exclusion statistics, we report partly analytical and partly numerical results on thermodynamic properties of assemblies of particles obeying fractional exclusion statistics. The effect of dimensionality is one focal point, the ratio $\mu / k_{B} T$ of chemical potential to thermal energy being obtained numerically as a function of a scaled particle density. Pair correlation functions are also presented as a function of the statistical parameter, with Friedel oscillations developing close to the fermion limit, for sufficiently large density.
\end{abstract}

DOI: 10.1103/PhysRevE.76.061123

PACS number(s): 05.30.Pr, 71.10.Pm

\section{INTRODUCTION}

Current interest in fractional statistics [1-3] is motivated by its possible relevance for fractional quantum Hall effect $[4,5]$ and high-temperature superconductivity [6]. In this context, an early application was made by Lea et al. $[7,8]$ to discuss the de Haas-van Alphen oscillatory orbital magnetism of a two-dimensional electron gas. Quite recently, it has been proposed that noise experiments in quantum Hall fluids might reveal the existence of elementary excitations obeying fractional statistics $[9,10]$.

Fractional exchange statistics [1-3] arises when the many-body wave function of a system of indistinguishable particles (dubbed anyons) is allowed to acquire an arbitrary phase $e^{i \alpha \pi}$ upon an adiabatic exchange process of two particles. Here, $\alpha$ is the so-called statistical parameter, interpolating between $\alpha=0$ (bosons) and $\alpha=1$ (fermions). Such an exchange produces a nontrivial phase only if the configuration space of the collection of particles under study possesses a multiply connected topological structure. Therefore, fractional exchange statistics is usually restricted to two spatial dimensions, $d=2$ (see Ref. [11] for a review). However, fractional exchange statistics can be formalized, to some extent, also in $d=1[12,13]$.

A different concept of fractional statistics, namely fractional exclusion statistics, is based on the structure of the Hilbert space, rather than the configuration space, of the particle assembly, and is therefore not restricted to $d \leq 2$. Fractional exclusion statistics has been introduced by Haldane [14], who considered the ratio

$$
g=-\Delta D / \Delta N,
$$

where $\Delta D$ denotes the change in size of the subset of available states in the Hilbert space corresponding to a change $\Delta N$ of the number of particles (i.e., elementary excitations). Clearly, one has again $g=0$ for bosons and $g=1$ for fermions, the latter being a consequence of Pauli exclusion principle.

\footnotetext{
*Corresponding author; giuseppe.angilella@ct.infn.it
}

At variance with anyons, particles obeying fractional exclusion statistics are usually dubbed $g$-ons or exclusons. The distribution function for fractional exclusion statistics has been derived by $\mathrm{Wu}$ [15]. A limiting form of the same distribution function had been derived by March et al. [16] within an approximate chemical collision model (see also Refs. [17,18]). Following Wu's distribution function [15], the thermodynamics of an ideal gas with fractional exclusion statistics has been studied in some detail in arbitrary dimensions $[19,20]$.

The relation between fractional exchange and exclusion statistics is elusive. In particular, it has been emphasized [21] that in order to derive a consistent statistical mechanics for $g$-ons, Haldane's generalized exclusion principle, Eq. (1), must hold locally in phase space, which indeed applies rigorously to either bosons or fermions. In other words, $\Delta D$ in Eq. (1) should be related to states of close energy, which is brought about by an effective interaction which is local in momentum space. From this, it has been concluded that anyons are not ideal $g$-ons, but interacting $g$-ons [21]. Haldane [14] provides explicit examples of systems for which fractional exclusion, albeit not exchange, statistics might be applicable. An earlier attempt to relate the parameter $g$ of fractional exclusion statistics and the statistical parameter $\alpha$ of fractional exchange statistics has been made in Ref. [22], on the basis of the virial expansion of the $g$-ons partition function. More recently, an analytic, monotonic relation $g=g(\alpha)$ has been derived in Ref. [23] in the case $g$ $=1 / \mathrm{m}$ (for integer $\mathrm{m}$ ), which is relevant for the fractional quantum Hall effect (see also Ref. [24] for a review).

In an electron gas, statistical correlations between noninteracting fermions arise as a consequence of particle indistinguishability. In the case of fermions, the antisymmetry of the wave function implies that two such particles can never be found simultaneously at the same point in space. As a result, each electron is surrounded by an exchange hole, a region in which the density of same-spin electrons is smaller than average and in which, therefore, the positive background charge is not exactly cancelled. It is the interaction of each electron with the positive charge of the exchange hole 
that gives rise to the exchange energy. The situation is quite the opposite for bosons, where statistical correlations are attractive and may be thought of being ultimately responsible of Bose-Einstein condensation. In the case of intermediate or fractional statistics, the question naturally arises of how many particles does a particle effectively "see" around itself. In other words, one is led to consider the effect of Haldane's generalized exclusion principle on the exchange hole which a $g$-on digs around itself.

In this work, we attempt to answer such a question, by evaluating a suitably defined generalization of the pair correlation function between $g$-ons (i.e., particles obeying fractional exclusion statistics), in arbitrary dimensions $d$ ( $d$ $=1,2,3)$, as a function of temperature $T$ and particle density. Earlier results along this direction include the seminal work of Sutherland [25], for a system of fermions or bosons in $d$ $=1$ interacting through a singular potential $\sim 1 / r^{2}$, a problem which can be mapped into anyons [11]. The evaluation of a pairwise correlation function for anyons (i.e., particles obeying fractional exchange statistics) in $d=2$ at $T=0$ has been also recently analyzed in Ref. [26], in connection with an intensity interferometry gedanken experiment, which may prove useful in the context of quantum computing. The question is also intimately related to that of finding Uhlenbeck's statistical interparticle potential [27], which has been studied for the two-anyons system in $d=2$ [28].

The paper is organized as follows. In Sec. II we derive the pair correlation function for a homogeneous assembly of $g$-ons as a function of interparticle separation $r$. In Sec. II A we study numerically the dependence of the chemical potential on density at a given temperature, for arbitrary dimensions $d=1,2,3$, thereby reproducing Wu's analytical result at $d=2$. In Sec. II B we study numerically the pair correlation function, and in particular focus on the possible occurrence of Friedel oscillations, depending on the statistical parameter and on the scaled density. We eventually summarize in Sec. III.

\section{PAIR CORRELATION FUNCTION FOR NONINTERACTING $g$-ONS}

Statistical correlations between indistinguishable, hardcore particles can be described by means of the pair correlation function $g(\mathbf{r})$, defined as the normalized probability of simultaneously finding a particle at position $\mathbf{r}$ and a particle at position $\mathbf{r}=0$. (Here and below, we are implicitly neglecting spin.) In the formalism of second quantization, the pair correlation function can be written as

$$
g(\mathbf{r})=\frac{\left\langle\Psi^{\dagger}(\mathbf{r}) \Psi^{\dagger}(0) \Psi(0) \Psi(\mathbf{r})\right\rangle}{n(\mathbf{r}) n(0)} .
$$

Here, $\Psi^{\dagger}(\mathbf{r})[\Psi(\mathbf{r})]$ is a creation (annihilation) quantum field operator at position $\mathbf{r}, n(\mathbf{r})=\left\langle\Psi^{\dagger}(\mathbf{r}) \Psi(\mathbf{r})\right\rangle$ is the singleparticle probability density at position $\mathbf{r}$, and $\langle\cdots\rangle$ denotes a quantum statistical average associated with the equilibrium distribution of the particle assembly under study (see below). In the case of a homogeneous system, one obviously has $n(\mathbf{r})=n(0)$ and $g(\mathbf{r}) \equiv g(r)$.
In the case of either fermions or bosons, the four-point average appearing in the right-hand side of Eq. (2) can be reduced by means of Wick's theorem as

$$
\begin{aligned}
& \left\langle\Psi^{\dagger}(\mathbf{r}) \Psi^{\dagger}(0) \Psi(0) \Psi(\mathbf{r})\right\rangle \\
& \quad=\left\langle\Psi^{\dagger}(\mathbf{r}) \Psi(\mathbf{r})\right\rangle\left\langle\Psi^{\dagger}(0) \Psi(0)\right\rangle \pm\left\langle\Psi^{\dagger}(\mathbf{r}) \Psi(0)\right\rangle\left\langle\Psi^{\dagger}(0) \Psi(\mathbf{r})\right\rangle,
\end{aligned}
$$

the plus (minus) sign corresponding to bosons (fermions). In the case of particles obeying exclusion statistics, the appropriate generalization of Wick's theorem depends on the behavior of exclusion field operators under exchange. As emphasized in the Introduction, the relation between fractional exclusion and exchange statistics is not completely settled. Within fractional exchange statistics in reduced dimensionality $(d \leq 2)$, one obviously has the following graded commutation relations:

$$
\begin{gathered}
{\left[\Psi(\mathbf{r}), \Psi\left(\mathbf{r}^{\prime}\right)\right]_{\alpha} \equiv \Psi(\mathbf{r}) \Psi\left(\mathbf{r}^{\prime}\right)-e^{i \alpha \pi s\left(\mathbf{r}, \mathbf{r}^{\prime}\right)} \Psi\left(\mathbf{r}^{\prime}\right) \Psi(\mathbf{r})=0,} \\
{\left[\Psi^{\dagger}(\mathbf{r}), \Psi^{\dagger}\left(\mathbf{r}^{\prime}\right)\right]_{\alpha}=0,} \\
{\left[\Psi(\mathbf{r}), \Psi^{\dagger}\left(\mathbf{r}^{\prime}\right)\right]_{\alpha}=\delta\left(\mathbf{r}-\mathbf{r}^{\prime}\right),}
\end{gathered}
$$

with $s\left(\mathbf{r}, \mathbf{r}^{\prime}\right)= \pm 1$, depending on whether the exchange is being performed along a (counter)clockwise path in $d=2$ [11], or $s\left(\mathbf{r}, \mathbf{r}^{\prime}\right)=\operatorname{sgn}\left(r-r^{\prime}\right)$ in $d=1$ [12]. Equations (4a)-(4c) naturally reduce to the familiar commutation and anticommutation relations for bosons $(\alpha=0)$ and fermions $(\alpha=1)$, respectively. In $d=3$, the sign $s\left(\mathbf{r}, \mathbf{r}^{\prime}\right)$ is ill-defined, and in fact fractional exchange statistics does not apply to dimensions $d \geq 3$. In order to have generalized commutation relations valid for exclusons in arbitrary dimensions, we therefore assume real field operators, assign a statistical exchange parameter $\alpha$ to exclusons ( $g$-ons), and postulate that standard quantum Bose or Fermi statistics are weakly violated as in

$$
\left[\Psi(\mathbf{r}), \Psi\left(\mathbf{r}^{\prime}\right)\right]_{\alpha} \equiv \Psi(\mathbf{r}) \Psi\left(\mathbf{r}^{\prime}\right)-\cos (\alpha \pi) \Psi\left(\mathbf{r}^{\prime}\right) \Psi(\mathbf{r})=0,
$$

$$
\begin{gathered}
{\left[\Psi^{\dagger}(\mathbf{r}), \Psi^{\dagger}\left(\mathbf{r}^{\prime}\right)\right]_{\alpha}=0,} \\
{\left[\Psi(\mathbf{r}), \Psi^{\dagger}\left(\mathbf{r}^{\prime}\right)\right]_{\alpha}=\delta\left(\mathbf{r}-\mathbf{r}^{\prime}\right),}
\end{gathered}
$$

which again reduce to the familiar relations for bosons and fermions, in the appropriate limits. Following Ref. [29] for the generalization of Wick's theorem corresponding to Eqs. (5a)-(5c), Eq. (3) becomes

$$
\begin{aligned}
&\left\langle\Psi^{\dagger}(\mathbf{r}) \Psi^{\dagger}(0) \Psi(0) \Psi(\mathbf{r})\right\rangle \\
&=\left\langle\Psi^{\dagger}(\mathbf{r}) \Psi(\mathbf{r})\right\rangle\left\langle\Psi^{\dagger}(0) \Psi(0)\right\rangle+\cos (\alpha \pi)\left\langle\Psi^{\dagger}(\mathbf{r}) \Psi(0)\right\rangle \\
& \times\left\langle\Psi^{\dagger}(0) \Psi(\mathbf{r})\right\rangle .
\end{aligned}
$$

Correspondingly, the pair correlation function for a homogeneous assembly of $g$-ons reads 


$$
g(r)=1+\cos (\alpha \pi) \frac{\left|\left\langle\Psi^{\dagger}(r) \Psi(0)\right\rangle\right|^{2}}{n^{2}(0)},
$$

which correctly reduces to the fermion limit $(\alpha=1)$, with $g(r) \leq 1$, manifestly [30].

For translationally invariant systems, one may use a plane wave expansion to find

$$
g(r)=1+\cos (\alpha \pi)\left|\frac{\widetilde{n}(r)}{\widetilde{n}(0)}\right|^{2},
$$

where

$$
\widetilde{n}(r)=\int \frac{d^{d} \mathbf{k}}{(2 \pi)^{d}} e^{-i \mathbf{k} \cdot \mathbf{r}} n(\mathbf{k})
$$

is the Fourier transform in $d$ dimensions of the single-particle distribution function $n(\mathbf{k})$ for $g$-ons in equilibrium at temperature $T$.

Following $\mathrm{Wu}$ [15], the average occupation number $n_{i}$ of exclusons in an energy state $\epsilon_{i}$ at equilibrium is

$$
n_{i}=\frac{1}{w(\zeta)+\alpha},
$$

where $\zeta=\exp \left[\left(\epsilon_{i}-\mu\right) / k_{B} T\right], \mu$ is the chemical potential, and the function $w(\zeta)$ satisfies the functional equation

$$
w^{\alpha}(1+w)^{1-\alpha}=\zeta .
$$

Here and below, we shall use the standard notation $g \equiv \alpha$, even for exclusons. Equation (10) establishes the thermodynamics of the excluson assembly, once the energy level distribution $\epsilon_{i}$ is given, which is determined by the free-particle dynamics. In order to make contact with the ordinary Bose and Fermi limits, we shall then assume that $\epsilon_{i}=\hbar^{2} k_{i}^{2} /(2 m)$, and then treat $\mathbf{k}_{i} \mapsto \mathbf{k}$ as a continuous variable, as is understood in Eq. (8) with $n_{i} \mapsto n(\mathbf{k})$.

An approximate form of the distribution function for exclusons had been earlier derived by March et al. [16-18] from collision theory, by using the detailed balance hypothesis. Their result was that $n_{i}^{-1}=\zeta+a$, with $a$ interpolating between Bose $(a=-1)$ and Fermi $(a=+1)$ statistics. Comparison with Wu's result, Eq. (10), then yields $[16,18]$

$$
a(\alpha, w)=w+\alpha-w^{\alpha}(1+w)^{1-\alpha} \approx 2 \alpha-1,
$$

the latter approximation, independent of $w$, holding in the limit $w \gg 1$. Equation (12) already correctly interpolates between the Bose $(\alpha=0, a=-1)$ and Fermi $(\alpha=1, a=1)$ limits. Of course, as $w$ becomes small, the dependence of $a$ on $w$ is significant, though $2 \alpha-1$ is not a bad approximation even at $w=1$ (giving for $\alpha=\frac{1}{2}, a=0$ instead of 0.09 , and for $\alpha$ $=\frac{1}{4}, a=-\frac{1}{2}$ rather than -0.44$)$.

\section{A. Chemical potential}

Within these assumptions, the general properties and some exact results of the thermodynamics of an assembly of noninteracting exclusons have been derived to some extent $[15,19,20]$. Some properties of Wu's function $w(\zeta)$ in the complex $\zeta$ plane and their relevance for Cooper pairing of

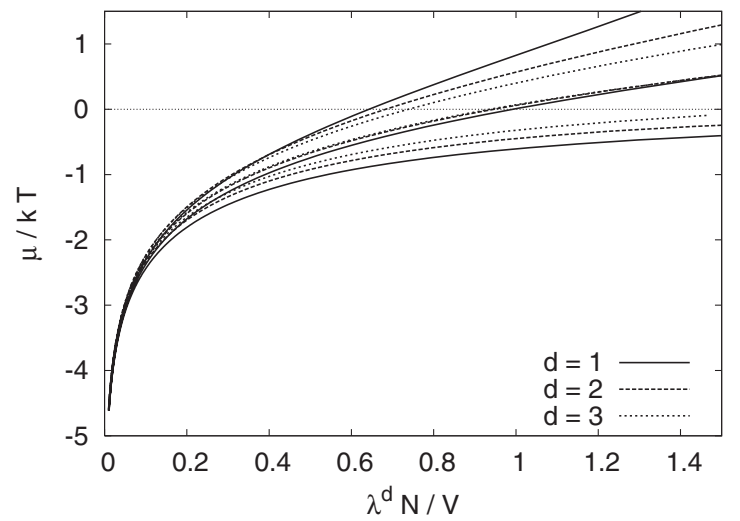

FIG. 1. Chemical potential vs scaled density, Eq. (15), for $\alpha$ $=0,0.5,1$ (bottom to top) and $d=1,2,3$.

exclusons have been discussed in Ref. [31]. In particular, by evaluating the particle density

$$
\frac{N}{V}=\int \frac{d^{d} \mathbf{k}}{(2 \pi)^{d}} n(\mathbf{k})
$$

in dimensions $d=2, \mathrm{Wu}[15]$ was able to derive the relation between chemical potential $\mu$, temperature $T$, and particle density $N / V$ explicitly as

$$
\frac{\mu}{k_{B} T}=\alpha \frac{\lambda^{2} N}{V}+\ln \left[1-\exp \left(-\frac{\lambda^{2} N}{V}\right)\right],
$$

where $\lambda=\hbar \sqrt{2 \pi /\left(m k_{B} T\right)}$ is the thermal wavelength. Performing the integration in Eq. (13) for a homogenous system in arbitrary dimensions $d$ (see also Ref. [20]), such a relation can be generalized implicitly as

$$
\Gamma\left(\frac{d}{2}\right) \frac{\lambda^{d} N}{V}=\int_{w_{0}}^{\infty} d w \frac{1}{w(1+w)} \bar{\epsilon}^{(d-2) / 2},
$$

where $\Gamma(x)$ is Euler's function, $w_{0} \equiv w_{0}\left(\mu / k_{B} T\right)$ is a generalized inverse fugacity, implicitly defined by Wu's functional equation for $\epsilon=0$, i.e.,

$$
w_{0}^{\alpha}\left(1+w_{0}\right)^{1-\alpha}=e^{-\mu / k_{B} T}
$$

and

$$
\bar{\epsilon}=\ln \left[w^{\alpha}(1+w)^{1-\alpha} e^{\mu / k_{B} T}\right] \equiv \ln \frac{w^{\alpha}(1+w)^{1-\alpha}}{w_{0}^{\alpha}\left(1+w_{0}\right)^{1-\alpha}} .
$$

Eliminating $w_{0}$ between Eqs. (15) and (16), we obtain the relation between the ratio $\mu / k_{B} T$ of chemical potential to thermal energy and the scaled density $\lambda^{d} N / V$, parametrically. This is plotted in Fig. 1 for several values of the statistical parameter $\alpha$, and $d=1,2,3$. We explicitly note that the dilute limit $\lambda^{d} N / V \ll 1$ corresponds to $w_{0} \gg 1\left(\mu / k_{B} T \rightarrow-\infty\right)$. We thus recover a monotonic relation, as expected, which for $\alpha=0$ (Bose limit) leads to an infinitesimally small negative chemical potential, in the limit of large density.

\section{B. Pair correlation function}

Following an analogous procedure, and performing the integration required in Eq. (9) for an isotropic system in $d$ 


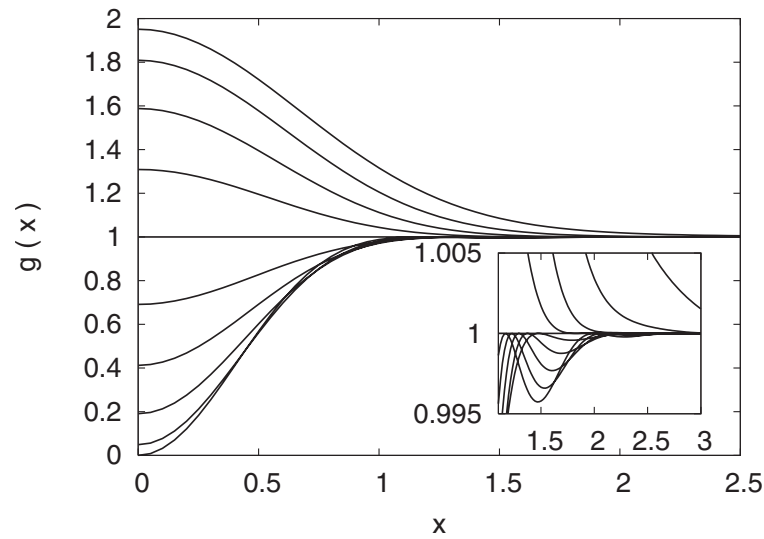

FIG. 2. Pair correlation function $g(x)$ (in scaled units), Eq. (8), in $d=2$, for $w_{0}=0.1$ (dense limit) and $\alpha=0-1$ (top to bottom). Inset shows Friedel oscillations, which are present only close to the Fermi distribution (FD) limit $(0.5<\alpha \leq 1)$.

dimensions, for the Fourier transform of the equilibrium distribution function of an assembly of noninteracting exclusons we find

$$
\lambda^{d} \tilde{n}(x)=x^{-\nu} \int_{w_{0}}^{\infty} d w \frac{1}{w(1+w)} \bar{\epsilon}^{\nu / 2} J_{\nu}\left(2 x \overline{\boldsymbol{\epsilon}}^{1 / 2}\right),
$$

where $x=\sqrt{\pi} r / \lambda, \nu=(d-2) / 2$, and $J_{\nu}(z)$ is a Bessel function of the first kind, arising from the integration over angles in $d$ dimensions. Making use of the asymptotic properties of the Bessel functions [32], it can be shown that Eq. (18) correctly reduces to Eq. (15) for the particle density in the limit $x$ $\rightarrow 0$. Further analytical results concerning the local excluson density $\widetilde{n}(x)$ are derived in the Appendix.

Figure 2 shows $g(x)$ in $d=2$ for $w_{0}=0.1$ (corresponding to a relatively large particle density), and for $\alpha=0-1$. In the fermion limit $(\alpha=1)$ and close to it $(0.5 \leq \alpha \leq 1)$, the pair correlation function exhibits a correlation hole around $x=0$, whose depth decreases with decreasing $\alpha$ and eventually vanishes as $\alpha \rightarrow \frac{1}{2}$. In the same range of values of the statistical parameter, $g(x)$ is characterized by damped Friedel oscillations (cf. inset of Fig. 2), which are more pronounced close to the fermion limit. These oscillations are absent within numerical accuracy in the prevalently bosonic range of the statistical parameter, $0 \leq \alpha \lesssim 0.5$, where the pair correlation function displays a monotonically decreasing behavior, with correlations piling up at $x=0$, in contrast with the fermion limit. Qualitatively similar results (not shown here) are obtained in $d=1$ and $d=3$.

Figure 3 shows again the pair correlation function in $d$ $=2$ for $\alpha=0-1$, but now for $w_{0}=0.5$, corresponding to a lower particle density. While the fermion side $(0.5 \leq \alpha \leq 1)$ is still characterized by a correlation hole, and the boson side displays a monotonic decrease of correlations, with a maximum at $x=0$, Friedel oscillations are now beyond graphical resolution even in the quasifermion regime (cf. inset of Fig. 3 ), as an effect of the reduced particle density. Again, qualitatively similar results obtain also in $d=1$ and $d=3$.

In order to make more quantitative the latter statement, we now inquire on the condition giving rise to Friedel oscil-

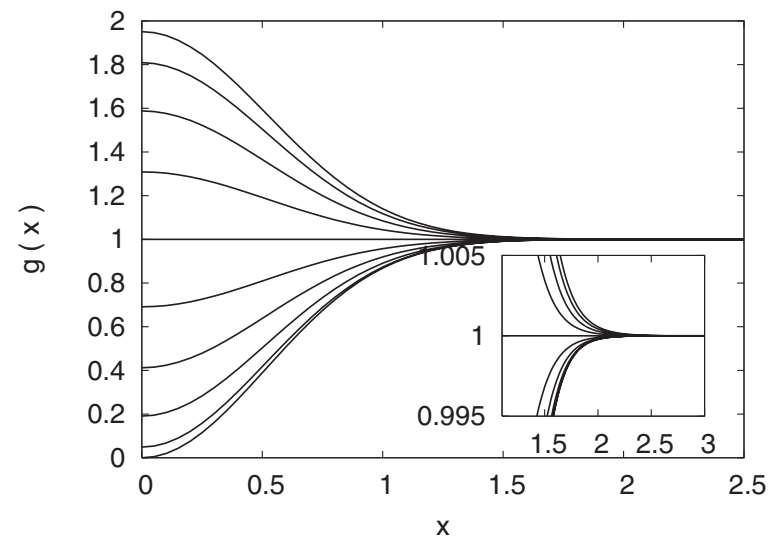

FIG. 3. Pair correlation function $g(x)$ (in scaled units), Eq. (8), in $d=2$, for $w_{0}=0.5$ (dilute limit) and $\alpha=0-1$ (top to bottom). Inset shows the absence of Friedel oscillations, even close to the Fermi distribution (FD) limit $(0.5 \leqq \alpha \leq 1)$.

lations in the pair correlation function. Generally speaking, Friedel oscillations in many-body properties of fermion assemblies arise from the presence of a discontinuity in the Fermi distribution function at the Fermi level. Besides decreasing at $T=0$ as a function of distance with a power law, depending on dimensionality, such oscillations are then exponentially damped with a characteristic decay length $\sim \lambda^{2} k_{F}$, with $k_{F}$ the Fermi wave vector.

In the case of particles obeying fractional exclusion statistics, Wu's distribution function, Eq. (10), in scaled units displays a smoother inflection point at a value $\bar{\epsilon}_{*}$ corresponding to $w_{*}=\alpha-1+\sqrt{\alpha^{2}-\alpha+1}$ in Eq. (17). When $w_{*} \geq w_{0}$, such an inflection point lies within the integration range in Eq. (18), and does therefore give rise to marked Friedel oscillations in the behavior of the pair correlation function. Such a condition has been studied numerically as a function of scaled particle density $\lambda^{d} N / V$ and statistical parameter $\alpha$, and is reproduced in Fig. 4 for various dimensionalities $d$ $=1,2,3$ in the form of a phase diagram.

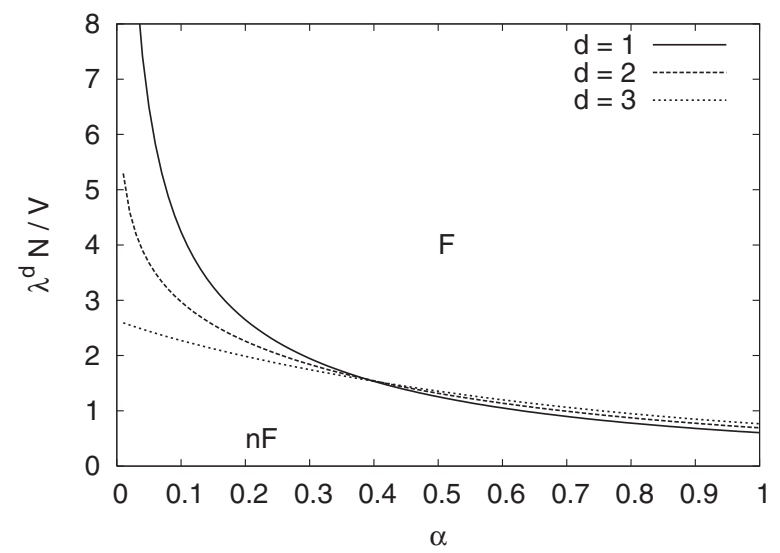

FIG. 4. Phase diagram in the plane of scaled density $\lambda^{d} N / V$ vs statistical parameter $\alpha$. The phase diagram features regions where pronounced Friedel oscillations occur in the pair correlation function $(F)$, and where such oscillations are absent, or beyond graphical resolution $(n F)$. Different lines refer to various dimensionalities $(d=1,2,3)$. 
The plane of scaled density $\lambda^{d} N / V$ vs statistical parameter $\alpha$ is divided into two regions by the line implicitly defined by the equation $w_{*}=w_{0}$. At a fixed value of the statistical parameter $\alpha$, say, one might think of increasing the fermioniclike correlations of a given $g$-on assembly (i.e., sharpening the Friedel oscillations in the pair correlation function) by suitably increasing its scaled density $\lambda^{d} N / V$, i.e., increasing density $N / V$ or reducing temperature $T$.

Within this picture, one may also estimate the wavelength $\Lambda$ of the oscillations in the pair correlation function with the approximate condition $2 \sqrt{\bar{\epsilon}_{*}} \Lambda \approx 2 \pi$, which straightforwardly leads to

$$
\Lambda^{2} \approx \frac{2 \pi^{2} \hbar^{2}}{m \epsilon_{*}},
$$

where all dimensions have been restored. Clearly, as $\epsilon_{*} \rightarrow 0$ as $\alpha \rightarrow 0$, one recovers the absence of Friedel oscillations in the bosonic limit.

\section{SUMMARY}

A brief discussion of the different concepts of fractional exchange and fractional exclusion statistics is first given. Then we have focused on some thermodynamical properties and on the pair correlation function of an assembly of noninteracting particles obeying exclusion statistics in arbitrary dimensionality $d=1,2,3$ and given temperature $T$. In particular, Eqs. (15) and (16) allow the chemical potential to be studied as a function of scaled particle density and dimensionality. The pair correlation function is then studied as a function of interparticle distance for different values of the statistical parameter $\alpha$ and of the scaled density $\lambda^{d} N / V$, where $\lambda$ is the de Broglie thermal wavelength. The behavior of the pair correlation function correctly exhibits a correlation hole in the fermion limit, and a pronounced maximum in the boson limit. Away from the boson limit, it also features Friedel oscillations, whose sharpness and wavelength can be tuned as a function of the statistical parameter and of scaled particle density. Our results show that, in principle, tuning density and temperature may sharpen the statistical correlations among noninteracting exclusons, thus ending in a behavior closer to either the fermionic or the bosonic limit, depending on dimensionality and on the value of the statistical parameter $\alpha$. This may be relevant to systems exhibiting the fractional quantum Hall effect [14], where anyons, i.e., particles obeying fractional exchange statistics, can be regarded as effectively interacting exclusons [21].

Note added in proof. An equivalent formulation of Eq. (11) has been independently derived by A. Dasnieres de Veigy and S. Ouvry $[33,34]$ for an anyon gas in a magnetic field. We thank Professor Ouvry for pointing out the above references.

\section{ACKNOWLEDGMENTS}

Two of the authors (G.G.N.A., N.H.M.) acknowledge that this paper was brought to completion during their stay at the Centro di Ricerca Matematica "Ennio De Giorgi" of the Scuola Normale Superiore, Pisa, Italy. Therefore they thank Professor M. P. Tosi and the Director of the Centre, Professor M. Giaquinta, for much hospitality and for the stimulating environment.

\section{APPENDIX: RECURSION FORMULAS FOR THE LOCAL EXCLUSON DENSITY}

Equation (18) yields $\widetilde{n}_{\nu}\left(x, w_{0}\right) \equiv \widetilde{n}(x)$ as a function of scaled interparticle distance $x$ and generalized inverse fugacity $w_{0}$, at a given statistical parameter $\alpha$ and reduced dimensionality $\nu=(d-2) / 2$. By differentiating Eq. (18) with respect to $x$, and making use of the appropriate recursion formula for the Bessel functions [32], one obtains

$$
\frac{\partial \widetilde{n}_{\nu}}{\partial x}+2 x \tilde{n}_{\nu+1}=0 .
$$

Similarly, by differentiating the same Eq. (18) with respect to $w_{0}$, and taking into account Eq. (17), one obtains

$$
\frac{\partial \widetilde{n}_{\nu}}{\partial w_{0}}+\left(\frac{d \bar{\epsilon}}{d w}\right)_{0} \tilde{n}_{\nu-1}+\rho_{\nu}\left(w_{0}\right)=0,
$$

where

$$
\left(\frac{d \bar{\epsilon}}{d w}\right)_{0}=\frac{\alpha+w_{0}}{w_{0}\left(1+w_{0}\right)}
$$

and

$$
\rho_{\nu}(w)=\frac{1}{\Gamma(\nu+1)} \frac{1}{w(1+w)} \bar{\epsilon}^{\nu}
$$

is the density of states in the $w$ variable, in $d=2 \nu+2$ dimensions. Equations (A1) and (A2) are then differential recursion formulas relating the local excluson density at different dimensionalities. Combining Eqs. (A1) and (A2), and noting that $\rho_{\nu+1}\left(w_{0}\right)=\lim _{w \rightarrow w_{0}} \rho_{\nu+1}(w)=0$ for $d=1,2,3$, one obtains

$$
\frac{\partial^{2} \widetilde{n}_{\nu}}{\partial w_{0} \partial x}=2 x \frac{\alpha+w_{0}}{w_{0}\left(1+w_{0}\right)} \widetilde{n}_{\nu}
$$

which is a hyperbolic partial differential equation for the local excluson density, similar to the Klein-Gordon equation, but with a variable mass term. 
[1] J. M. Leinaas and J. Myrheim, Nuovo Cimento Soc. Ital. Fis., B 37, 1 (1977).

[2] F. Wilczek, Phys. Rev. Lett. 48, 1144 (1982).

[3] F. Wilczek, Phys. Rev. Lett. 49, 957 (1982).

[4] R. B. Laughlin, Phys. Rev. Lett. 50, 1395 (1983).

[5] R. B. Laughlin, Phys. Rev. B 27, 3383 (1983).

[6] R. B. Laughlin, Phys. Rev. Lett. 60, 2677 (1988); 61, 379(E) (1988).

[7] M. J. Lea, N. H. March, and W. Sung, J. Phys.: Condens. Matter 3, 4301 (1991).

[8] M. J. Lea, N. H. March, and W. Sung, J. Phys.: Condens. Matter 4, 5263 (1992).

[9] E.-A. Kim, M. Lawler, S. Vishveshwara, and E. Fradkin, Phys. Rev. Lett. 95, 176402 (2005).

[10] F. E. Camino, W. Zhou, and V. J. Goldman, Phys. Rev. B 72, 075342 (2005).

[11] S. Forte, Rev. Mod. Phys. 64, 193 (1992).

[12] Z. N. C. Ha, Quantum Many-Body Systems in One Dimension (World Scientific, Singapore, 1996).

[13] P. Calabrese and M. Mintchev, Phys. Rev. B 75, 233104 (2007).

[14] F. D. M. Haldane, Phys. Rev. Lett. 67, 937 (1991).

[15] Y. S. Wu, Phys. Rev. Lett. 73, 922 (1994); 74, 3906(E) (1995).

[16] N. H. March, N. Gidopoulos, A. K. Theophilou, M. J. Lea, and W. Sung, Phys. Chem. Liq. 26, 135 (1993).
[17] N. H. March, J. Phys.: Condens. Matter 5, B149 (1993).

[18] N. H. March, Phys. Chem. Liq. 34, 61 (1997).

[19] G. S. Joyce, S. Sarkar, J. Spałek, and K. Byczuk, Phys. Rev. B 53, 990 (1996).

[20] K. Iguchi, Phys. Rev. Lett. 78, 3233 (1997).

[21] C. Nayak and F. Wilczek, Phys. Rev. Lett. 73, 2740 (1994).

[22] M. V. N. Murthy and R. Shankar, Phys. Rev. Lett. 72, 3629 (1994).

[23] A. D. Speliotopoulos, J. Phys. A 30, 6177 (1997).

[24] G. S. Canright and M. D. Johnson, J. Phys. A 27, 3579 (1994).

[25] B. Sutherland, J. Math. Phys. 12, 246 (1971).

[26] T. D. Gutierrez, Phys. Rev. A 69, 063614 (2004).

[27] G. E. Uhlenbeck and L. Gropper, Phys. Rev. 41, 79 (1932).

[28] W.-H. Huang, Phys. Rev. B 52, 15090 (1995).

[29] O. W. Greenberg, Phys. Rev. D 43, 4111 (1991).

[30] G. Giuliani and G. Vignale, Quantum Theory of the Electron Liquid (Cambridge University Press, Cambridge, 2005).

[31] G. G. N. Angilella, N. H. March, F. Siringo, and R. Pucci, Phys. Chem. Liq. 44, 343 (2006).

[32] I. S. Gradshteyn and I. M. Ryzhik, Table of Integrals, Series, and Products, 5th ed. (Academic, Boston, 1994).

[33] A. Dasnieres de Veigy and S. Ouvryde, Phys. Rev. Lett. 72, 600 (1994).

[34] A. Dasnieres de Veigy and S. Ouvryde, Mod. Phys. Lett. B 9, 271 (1995). 